\title{
Reliability Analysis for Degradation Data Based on Stochastic Process Models and Bayesian Estimations
}

\author{
Toru Kaise \\ Graduate School of Business, University of Hyogo \\ 8-2-1 Gakuen-Nishimachi, Nishiku, Kobe 651-2197, Japan \\ E-mail: kaise@mba.u-hyogo.ac.jp
}

\begin{abstract}
The Lévy process is considered to predict reliability assessments for degradation phenomena. In particular, the geometric Brownian motion and the gamma process models are handled, and the Bayesian procedures are applied to the models. The Markov chain Monte Carlo method and the empirical Bayesian method based on hierarchical Bayesian modeling are used to estimate the parameters. In addition, the maximum likelihood method and the generalized moment method are also appropriated to the models. The information criterion EIC is used to select a fitted model among the stochastic models and the estimation methods. The marginal likelihood with the Laplace's method plays important roles in the methodologies proposed in this paper.
\end{abstract}

\section{Introduction}

Infrastructures depend on many industrial items. Therefore defections of the items cause grave failures. Reliability problems are included in many areas of the engineering discipline, and many methodologies for the achievement of high reliability are developed. In particular, degradation phenomena have been treated as significant causes of many accidents. Stochastic methods of failure predicting based on strength and fatigues of materials play important roles for preventions of failures.

The physical phenomena of degradation are influenced by dynamical environments of the items. The surroundings generate random factors affecting to the degradation of the items, and then it is assumed that the degradation processes are treated by diffusion models. In references, various stochastic process models are applied to the degradation phenomena[1],[2]. The Brownian motion, the geometric Brownian motion, the gamma process are used for the phenomena, and accelerated models also are consisted with the diffusion models[3],[4],[5]. The maximum likelihood method is used to estimate the parameters of the stochastic process models, and the model selection is implemented by the information criterion AIC. The Bayesian estimation is also used in the reliability analysis for stress and strength models[6].
In this paper, the Lévy process is considered as the origin of the stochastic process models for the degradation phenomena. The geometric Brownian motion, the gamma process model and jump descriptions are handled based on the Bayesian and the hierarchical Bayesian methods. The information criterion EIC is applied to the models with the Bayesian methodologies [7], and it is shown that a comparison between these and the models with other estimation methods is possible. In particular, the maximum likelihood and the generalized moment method are applied to the models for the comparison. Therefore, it is shown that the reliability analysis for degradation phenomena is effective due to the Lévy processes by through the theoretical construction.

The rest of the paper is outlined as follows. Section 2 describes the models based on the Lévy process. In section 3, the estimation methods are explained and the Bayesian methods with the Markov chain Monte Carlo and the Laplace's methods based on the stochastic models are proposed. In addition, the information criterion EIC is described and the application to the stochastic models and the estimation methods is discussed.

\section{Stochastic Models}

\subsection{Damage and Degradation Modeling}

Let $X_{s}$ be a state of the degradation at some time $s$, and it assumed that the cumulative damage at $X_{s}$ is given by a function $c\left(X_{s}\right)$. Let be a continuous damage $W_{s}$, then the damage is described by the following derivative form

$$
d c\left(X_{s}\right)=h\left(X_{s}\right) d W_{s},
$$

where $h(\cdot)$ is a function of the damage modeling [5]. The form (1) means that the damage $W_{s}$ causes the degradation phenomena $c\left(X_{s}\right)$ with conditional the state $X_{s}$. Let $W_{s}$ be a stochastic process, then the form of the relation (1) is described by the following stochastic integration

$$
\int_{0}^{t} \frac{1}{h\left(X_{s}\right)} d c\left(X_{s}\right)=\int_{0}^{t} d W_{s}
$$


where $X_{s}$ is a stochastic process. For example, if $c\left(X_{s}\right)=X_{s}, h\left(X_{s}\right)=1$ and $W_{s}$ is a the Brownian motion, then $X_{s}$ is a Gaussian process, and in the case of $c\left(X_{s}\right)=\log X_{s}, X_{s}$ is the geometric Brownian motion. The Brownian motion $W_{s}$ means $W_{s}=0$ and it is given by the normal distribution $N(0, s)$ with mean 0 and variances.

In fact, it is difficult to observe the degradation state $X_{s}$, and the observation data are results of the degradation. In the case of the strength of materials, the observation data are fatigue cracks. The fatigue is caused by the degradation which is occurred by the dislocation of the cell structure and the slip of the face. Therefore the degradation could not be observed. The filtering methods for the state estimation might be applied to the problems. However in this paper, it is assumed that the clack is the degradation of the materials. We assume that the failure of a material occurred by the threshold of a damage point $K$ for the clack. Fig. 1 shows the abstract of the degradation modeling.

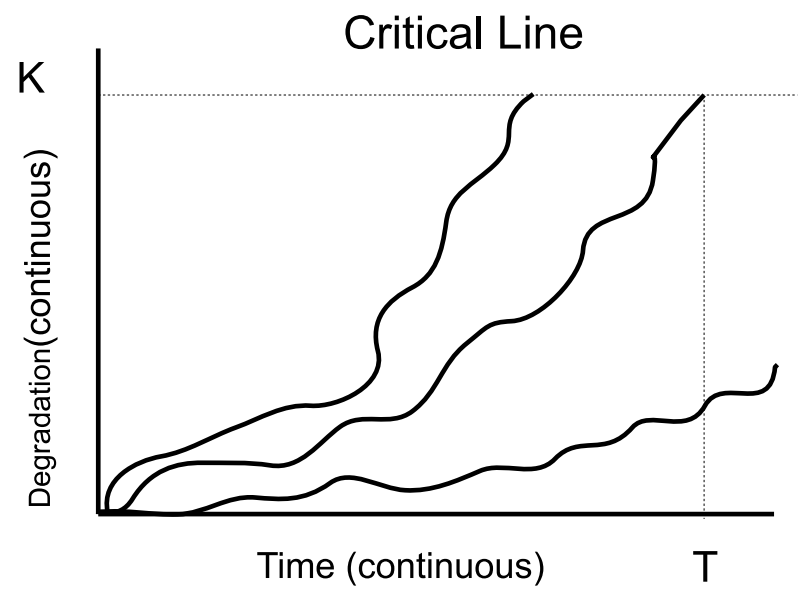

Fig. 1: The figure of the abstract for the degradation modeling.

\subsection{Lévy process}

Let $X_{t}$ be a random variable of the stochastic process, where $t$ is a continuous number. In particular let $x$ be an actual number of the stochastic process with ellipsis of $t$. The characteristic function $\psi(u)$ of the Lévy process is given by

$$
\begin{array}{r}
\psi(u)=\exp \left[-\frac{\sigma^{2}}{2} u^{2}+\int_{|X|<1}\left(e^{i u x}-1-i u x\right) \nu(d x)\right. \\
\left.+\int_{|X| \geq 1}\left(e^{i u x}-1\right) \nu(d x)+i b u\right],
\end{array}
$$

where $\sigma^{2} \in \Re_{+}, b \in \Re$ and $\nu(d x)$ is a measure on $\Re$ satisfying $\nu(0)=0, \int_{R}\left(1 \wedge x^{2}\right) \nu(d x)<\infty$. The Brownian motion, the gamma process and the compound Poisson process etc. are generated by the Lévy process. The Brownian motion is given by the measure $\nu(d x)=0$, and the geometric Brownian motion is generated by the translation of the exponential function for the Brownian motion. The gamma process is an increasing process with the measure $\nu(d x)=\alpha x^{-1} \exp (-\beta x) d x$, where $\alpha$ and $\beta$ are non-negative real numbers.

\subsection{Degradation Process Models}

The geometric Brownian motion is described by the following stochastic differential equation

$$
d X_{t}=b\left(X_{t}, \boldsymbol{\theta}\right) d t+\sigma\left(X_{t}, \boldsymbol{\theta}\right) d W_{t},
$$

where $\boldsymbol{\theta}=\left[\theta_{1}, \theta_{2}\right]^{T}$ is a parameter vector, $b(\cdot)$ and $\sigma(\cdot)$ are functions. In this case, $b\left(X_{t}, \boldsymbol{\theta}\right)=\theta_{1} X_{t}$ and $\sigma\left(X_{t}, \boldsymbol{\theta}\right)=\theta_{2} X_{t}, X_{0}=x_{0}, \theta_{1} \in \Re, \theta_{2} \in \Re_{+}$. The conditional probability density function $f_{\boldsymbol{\theta}}(t, y \mid x)$ is given by

$$
\begin{aligned}
& f_{\boldsymbol{\theta}}(t, y \mid x)= \\
& \frac{1}{y \theta_{2} \sqrt{2 \pi t}} \exp \left[-\frac{\left(\log y-\left(\log x+\left(\theta_{1}-\frac{1}{2} \theta_{2}^{2}\right) t\right)\right)^{2}}{2 \theta_{2}^{2} t}\right],
\end{aligned}
$$

where $x$ and $y$ are actual numbers of the random variables for the stochastic process. The random variable of the minimum reach time is denoted by $S$, which means $S=\inf \left\{t \mid X_{t} \leq K\right\}$. The probability function of $S$, which is denoted by $g_{S}\left(s \mid x_{0}, K\right)$, is given by

$$
g_{S}\left(s \mid x_{0}, K\right)=\frac{\sqrt{\lambda}}{\sqrt{2 \pi s^{3}}} \exp \left[-\frac{\lambda(s-\xi)^{2}}{2 \xi^{2} s}\right]
$$

where it is the inversed normal distribution, and $\xi=$ $\left(\log K-\log x_{0}\right) /\left(\theta_{1}-1 / 2 \theta_{2}^{2}\right), \lambda=\left(\log K-\log x_{0}\right)^{2} / \theta_{2}^{2}$.

The gamma process is generated by the Lévy process, and its stochastic differential equation is given by

$$
d X_{t}=b\left(X_{t}, \boldsymbol{\theta}\right) d t+\sigma\left(X_{t}, \boldsymbol{\theta}\right) d \gamma_{t},
$$

where $d \gamma_{t}$ satisfies $\gamma_{0}=0$, and if $t>s \geq 0$ then $\left(\gamma_{t}-\gamma_{s}\right)$ has the probability density function $g(u)$ which is given by

$$
g(u)=\frac{u^{h-1}}{\Gamma(h)} \exp [-u], h=t-s
$$

where it is the gamma distribution $G a(1, h)[1]$. When $d \gamma_{t}$ is substituted for the Wiener process $d W_{t}$, and $b\left(X_{t}, \boldsymbol{\theta}\right)=\theta_{1}\left(\theta_{2}-X_{t}\right), \theta_{1} \in \Re_{+}, \theta_{2} \in \Re_{+}, \sigma\left(X_{t}, \boldsymbol{\theta}\right)=$ $\sqrt{\theta_{3} X_{t}}, \theta_{3} \in \Re_{+}, X_{t}$ is generated by the gamma process [8]. The gamma process is defined as

1. $X_{0}=x_{0}\left(x_{0}=0\right)$;

2. $X_{t}-X_{s} \sim G a\left(\theta_{1}, h \theta_{2}\right)$;

3. $X_{t}$ has identical increments.

The property as the identical increments of the gamma process is appropriate to the description for the degradation. The conditional probability density function $f_{\boldsymbol{\theta}}(t, y \mid x)$ of $G a\left(\theta_{1}, h \theta_{2}\right)$ is given by

$f_{\boldsymbol{\theta}}(t, y \mid x)=\left(\frac{1}{\theta_{1}}\right)^{t \theta_{2}}(y-x)^{t \theta_{2}-1} \frac{1}{\Gamma\left(t \theta_{2}\right)} \exp \left[-\frac{(y-x)}{\theta_{1}}\right]$, 
where $\theta_{1} \in \Re_{+}, \theta_{2} \in \Re_{+}$. In addition, $y$ and $x$ means the stochastic processes, moreover $t$ is the interval time of the deference between $y$ and $x$. The random variable of the minimum reach time $S$ means $S=\inf \left\{t \mid X_{t} \leq\right.$ $K\}$, and the probability density function $g_{S}\left(s \mid x_{0}, K\right)$ is given by

$$
\begin{aligned}
& g_{S}\left(s \mid x_{0}, K\right)= \\
& \theta_{2}\left\{\Psi\left(\theta_{2} s\right)-\log K_{\theta_{1}}\right\}\left\{1-\frac{\gamma\left(\theta_{2} s, K_{\theta_{1}}\right.}{\gamma\left(\theta_{2} s\right)}\right\} \\
& +\frac{\theta_{2}}{\gamma\left(\theta_{2} s\right)} \frac{K_{\theta_{1}}^{\theta_{2} s}}{\left(\theta_{2} s\right)^{2}}\left\{1+\sum_{j=1}^{\infty}\left(\frac{\theta_{2} s}{\theta_{2} s+j}\right)^{2} \frac{K_{\theta_{1}}^{j}}{j !}\right\},
\end{aligned}
$$

where $K_{\theta_{1}}=\left(K-x_{0}\right) / \theta_{1}$, and $\Psi(z)=d / d z \log \gamma(z)$.

\section{Estimation Methods}

\subsection{Maximum Likelihood Estimation}

Let $L_{N}(\boldsymbol{\theta})$ be a likelihood function for the stochastic process models, and it is given by

$L_{N}(\boldsymbol{\theta})=\exp \left(\int_{0}^{N} \frac{b\left(X_{t}, \boldsymbol{\theta}\right)}{\sigma^{2}\left(X_{t}\right)} d X_{t}-\frac{1}{2} \frac{b^{2}\left(X_{t}, \boldsymbol{\theta}\right)}{\sigma^{2}\left(X_{t}\right)} d t\right)$,

where the continuous time interval is $[0, N][8]$. The process is observed at discrete times, therefore let $L_{n}(\boldsymbol{\theta})$ be a discrete counterpart of $L_{N}(\boldsymbol{\theta})$, it is given by

$$
L_{n}(\boldsymbol{\theta})=\prod_{i}^{n} f_{\boldsymbol{\theta}}\left(\Delta_{i}, X_{i} \mid X_{i-1}\right) f_{\boldsymbol{\theta}}\left(X_{0}\right)
$$

where $t_{i}=i \Delta_{i}, i=1,2, \ldots, n$ and $t_{i}$ is a discrete time shared from the continuous time, and it is assumed that $f_{\boldsymbol{\theta}}\left(X_{0}\right)=1$ and $n \Delta_{n}^{k} \rightarrow 0$ for some power $k \geq 2$. Let $D_{\boldsymbol{X}_{\boldsymbol{t}}}$ be a set of the data, and $l\left(\boldsymbol{\theta} \mid D_{\boldsymbol{X}_{\boldsymbol{t}}}\right)$ is the likelihood substituted for $L_{n}(\boldsymbol{\theta})$ based on $D_{\boldsymbol{X}_{\boldsymbol{t}}}$. The maximum likelihood estimate $\hat{\boldsymbol{\theta}}$ is derived from $\operatorname{argmax}_{\boldsymbol{\theta}} \log l\left(\boldsymbol{\theta} \mid D_{\boldsymbol{X}_{\boldsymbol{t}}}\right)$.

The maximum likelihood estimator has asymptotic properties which are the consistency, the efficiency. The Fisher information is denoted by $I(\boldsymbol{\theta})$, the variance of the estimator is given by $I^{-1}(\boldsymbol{\theta})$. Where $I(\boldsymbol{\theta})$ is given by

$$
\begin{aligned}
I(\boldsymbol{\theta}) & =E\left[\frac{\partial \log l\left(\boldsymbol{\theta} \mid D_{\boldsymbol{X}_{\boldsymbol{t}}}\right)}{\partial \boldsymbol{\theta}} \frac{\partial \log l\left(\boldsymbol{\theta} \mid D_{\boldsymbol{X}_{\boldsymbol{t}}}\right)}{\partial \boldsymbol{\theta}^{T}}\right] \\
& =E\left[-\frac{\partial^{2} \log l\left(\boldsymbol{\theta} \mid D_{\boldsymbol{X}_{\boldsymbol{t}}}\right)}{\partial \boldsymbol{\theta} \partial \boldsymbol{\theta}^{T}}\right]
\end{aligned}
$$

and the approximation of $I^{-1}(\boldsymbol{\theta})$ is denoted by
$i\left(\hat{\boldsymbol{\theta}}\left(\boldsymbol{X}_{\boldsymbol{t}}\right)\right)$, it is given by

$$
\begin{aligned}
& i\left(\hat{\boldsymbol{\theta}}\left(\boldsymbol{X}_{\boldsymbol{t}}\right)\right) \\
= & \left.\left(\frac{\partial \log l\left(\boldsymbol{\theta} \mid D_{\boldsymbol{X}_{\boldsymbol{t}}}\right)}{\partial \boldsymbol{\theta}} \frac{\partial \log l\left(\boldsymbol{\theta} \mid D_{\boldsymbol{X}_{\boldsymbol{t}}}\right)}{\partial \boldsymbol{\theta}^{T}}\right)\right|_{\boldsymbol{\theta}=\hat{\boldsymbol{\theta}}\left(\boldsymbol{X}_{\boldsymbol{t}}\right)} \\
= & -\left.\frac{\partial^{2} \log l\left(\boldsymbol{\theta} \mid D_{\boldsymbol{X}_{\boldsymbol{t}}}\right)}{\partial \boldsymbol{\theta} \partial \boldsymbol{\theta}^{T}}\right|_{\boldsymbol{\theta}=\hat{\boldsymbol{\theta}}_{\left(\boldsymbol{X}_{\boldsymbol{t}}\right)} .}
\end{aligned}
$$

\subsection{Generalized Moment Method}

When the moments of the stochastic processes $X_{i}$, $i=1,2 \cdots, n$ are provided, the generalized moment method (GMM) is adapted to estimate the parameters for the stochastic process model. When

$$
\begin{gathered}
u_{i}(\boldsymbol{\theta})=\left[\begin{array}{c}
X_{i+1}-E\left\{X_{i+1} \mid \mathcal{F}_{i}\right\} \\
X_{i}\left(X_{i+1}-E\left\{X_{i+1} \mid \mathcal{F}_{i}\right\}\right) \\
V\left\{X_{i+1} \mid \mathcal{F}_{i}\right\}-\left(X_{i+1}-E\left\{X_{i+1} \mid \mathcal{F}_{i}\right\}\right)^{2} \\
X_{i}\left\{V\left\{X_{i+1} \mid \mathcal{F}_{i}\right\}-\left(X_{i+1}-E\left\{X_{i+1} \mid \mathcal{F}_{i}\right\}\right)^{2}\right\}
\end{array}\right], \\
g_{n}(\boldsymbol{\theta})=\frac{1}{n} \sum_{i}^{n} u_{i}(\boldsymbol{\theta}),
\end{gathered}
$$

is given, the estimate of GMM which is denoted by $\hat{\boldsymbol{\theta}}\left(\boldsymbol{X}_{\boldsymbol{t}}\right)_{G M M}$ is derived by $\arg \min _{\boldsymbol{\theta}} g_{n}(\boldsymbol{\theta})^{T} W g_{n}(\boldsymbol{\theta})$, where $\mathcal{F}_{i}$ means the information system until $i, W=$ $E\left\{\boldsymbol{u} \boldsymbol{u}^{T}\right\}^{-1}$ and $\boldsymbol{u}=\left[u_{i}(\boldsymbol{\theta})\right], i=1,2, \cdots, n$.

\subsection{Bayesian Estimation and MCMC}

The Bayesian method offers the posterior distribution $p\left(\boldsymbol{\theta} \mid D_{\boldsymbol{X}_{\boldsymbol{t}}}\right)$ which is derived by

$$
p\left(\boldsymbol{\theta} \mid D_{\boldsymbol{X}_{\boldsymbol{t}}}\right) \propto l\left(\boldsymbol{\theta} \mid D_{\boldsymbol{X}_{\boldsymbol{t}}}\right) \pi(\boldsymbol{\theta}),
$$

where $\pi(\boldsymbol{\theta})$ is a prior distribution. In the case of the geometric Brownian motion, the prior distribution is considered as $\pi(\boldsymbol{\theta})=\pi\left(\theta_{1}\right) \pi_{2}\left(\theta_{2}\right)$, where $\pi\left(\theta_{1}\right)$ and $\pi_{2}\left(\theta_{2}\right)$ are given by

$$
\begin{aligned}
\pi\left(\theta_{1}\right) & =\frac{1}{2 \pi \sigma^{2}} \exp \left[-\frac{\left(\theta_{1}-\mu\right)^{2}}{2 \sigma^{2}}\right], \mu \in \Re, \sigma \in \Re_{+}, \\
\pi_{2}\left(\theta_{2}\right) & =\frac{b^{a}}{\gamma(a)} \theta_{2}^{a-1} \exp \left[-b \theta_{2}\right], a \in \Re_{+}, b \in \Re_{+},
\end{aligned}
$$

respectively. In the case of the gamma process, the prior distributions are given by

$$
\begin{aligned}
\pi\left(\theta_{1}\right) & =\frac{\beta^{\alpha}}{\gamma(\alpha)}\left(\frac{1}{\theta_{1}}\right)^{\alpha+1} \exp \left[-\frac{\beta}{\theta_{1}}\right], \alpha \in \Re_{+}, \beta \in \Re_{+}, \\
\pi_{2}\left(\theta_{2}\right) & =\frac{1}{\nu}, \quad \nu \in \Re_{+} .
\end{aligned}
$$

The Markov chain Monte Carlo (MCMC) method is used to estimate the posterior distribution. The posterior samples of the Gibbs sampling are provided by the Monte Carlo method based on the conditional posterior distribution, and the repeating method is represented 
by the following.

$$
\begin{aligned}
& \theta_{2}^{(0)} \sim \pi\left(\theta_{2}\right) \\
& i=1,2, \cdots, M, \\
& \theta_{1}^{(i)} \sim p\left(\theta_{1}^{(i)} \mid \theta_{2}^{(i-1)}, D_{\boldsymbol{X}_{\boldsymbol{t}}}\right) \\
& \theta_{2}^{(i)} \sim p\left(\theta_{2}^{(i)} \mid \theta_{1}^{(i)}, D_{\boldsymbol{X}_{\boldsymbol{t}}}\right) .
\end{aligned}
$$

The initial samples $i=1,2, \cdots, M_{o}$ are rejected as the burn-in period and the remainders of the samples are denoted by the posterior samples of $p\left(\theta_{1}, \theta_{2} \mid D_{\boldsymbol{X}_{\boldsymbol{t}}}\right)$. The posterior estimates which are denoted by $\hat{\theta}_{1 M C M C}$ and $\hat{\theta}_{2 M C M C}$ are derived by $\hat{\theta}_{1 M C M C}=\sum_{i=M_{o}+1}^{M} \theta_{1}^{(i)} /\left(M-M_{o}\right)$ and $\hat{\theta}_{2 M C M C}=$ $\sum_{i=M_{o}+1}^{M} \theta_{2}^{(i)} /\left(M-M_{o}\right)$.

In the Gibbs sampling method, the posterior samples are given easily by the Monte Carlo simulation based on the conditional posterior distributions. However in many cases, substitutions of the the conditional posteriors are seeked, because it is difficult to calculate numerically the simulation sampling based on the conditional posteriors. The Metropolis-Hasting method uses the substitute distributions and the repeat ions $f$ the Gibbs sampling. In MH method, $\theta^{\sharp}$ is generated by the substitute distribution $q\left(\theta, \theta^{\sharp}\right)$ with $\theta=\theta_{1}^{(i-1)}$. When $p\left(\theta \mid \theta_{2}^{(i-1)}, D_{\boldsymbol{X}_{\boldsymbol{t}}}\right) q\left(\theta, \theta^{\sharp}\right)>0$, it is adopted that

$$
\alpha\left(\theta, \theta^{\sharp}\right)=\min \left(\frac{p\left(\theta^{\sharp} \mid \theta_{2}^{(i-1)}, D_{\boldsymbol{X}_{\boldsymbol{t}}}\right) q\left(\theta^{\sharp}, \theta\right)}{p\left(\theta \mid \theta_{2}^{(i-1)}, D_{\boldsymbol{X}_{\boldsymbol{t}}}\right) q\left(\theta, \theta^{\sharp}\right)}, 1\right),
$$

however, when $p\left(\theta \mid \theta_{2}^{(i-1)}, D_{\boldsymbol{X}_{\boldsymbol{t}}}\right) q\left(\theta, \theta^{\sharp}\right)=0$, it is adopted that

$$
\alpha\left(\theta, \theta^{\sharp}\right)=1 .
$$

In next, a unified random number $u$ is given, and if $\alpha\left(\theta, \theta^{\sharp}\right)<u$, then $\theta^{\sharp}=\theta_{1}^{(i)}$. The same way is used for $\theta_{2}^{(i)}$. These handling processes are repeated as the Gibbs sampling.

In this paper, the normal distribution is applied to $q\left(\theta, \theta^{\sharp}\right)$, where the mean is the posterior mode $\hat{\boldsymbol{\theta}}\left(\boldsymbol{X}_{\boldsymbol{t}}\right)_{B M O}$ of the posterior distribution $p\left(\theta_{1}, \theta_{2} \mid D_{\boldsymbol{X}_{\boldsymbol{t}}}\right)$, and the variance is $i\left(\hat{\boldsymbol{\theta}}\left(\boldsymbol{X}_{\boldsymbol{t}}\right)_{B M O}\right)^{-1}$ based on the Fisher information with the mode $\hat{\boldsymbol{\theta}}\left(\boldsymbol{X}_{\boldsymbol{t}}\right)_{B M O}$.

\subsection{Empirical Bayes}

The hierarchical Bayesian method means usage of the marginal likelihood $l_{m}\left(\boldsymbol{\theta}_{h} \mid D_{\boldsymbol{X}_{\boldsymbol{t}}}\right)$ which is given by

$$
\begin{aligned}
l_{m}\left(\boldsymbol{\theta}_{h} \mid D_{\left.\boldsymbol{X}_{\boldsymbol{t}}\right)=}\right. & \int_{\Theta} l\left(\boldsymbol{\theta} \mid D_{\boldsymbol{X}_{\boldsymbol{t}}}\right) \pi\left(\boldsymbol{\theta} \mid \boldsymbol{\theta}_{h}\right) d \boldsymbol{\theta} \\
\approx & l\left(\hat{\boldsymbol{\theta}}\left(\boldsymbol{X}_{\boldsymbol{t}}\right)_{B M O} \mid D_{\boldsymbol{t}}\right) \pi\left(\hat{\boldsymbol{\theta}}\left(\boldsymbol{X}_{\boldsymbol{t}}\right)_{B M O} \mid \boldsymbol{\theta}_{h}\right) \\
& \times \frac{(2 \pi)^{\frac{V}{2}}}{n^{\frac{V}{2}}\left|i\left(\hat{\boldsymbol{\theta}}\left(\boldsymbol{X}_{\boldsymbol{t}}\right)_{B M O}\right)\right|^{\frac{1}{2}}},
\end{aligned}
$$

where $\boldsymbol{\theta}_{h}$ is the hyperparameter [9]. The Laplace's method for the integration is appropriated based on the posterior mode $\hat{\boldsymbol{\theta}}\left(\boldsymbol{X}_{\boldsymbol{t}}\right)_{B M O}$, where $V$ is the dimension of $\boldsymbol{\theta}$ and $i\left(\hat{\boldsymbol{\theta}}\left(\boldsymbol{X}_{\boldsymbol{t}}\right)_{B M O}\right)$ is given by

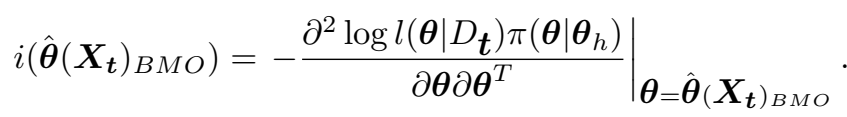

The jump is assumed by the prior distribution $\pi\left(\theta_{2}\right)$ and $\theta_{1}$ has no prior. The parameters $\boldsymbol{\theta}_{h}$ and $\theta_{1}$ are estimated by the maximization of $l_{m}\left(\boldsymbol{\theta}_{h} \mid D_{\boldsymbol{X}_{t}}\right)$. The geometric Brownian motion and the gamma process models are handled by this hierarchical Bayesian method.

\section{$3.5 \quad$ EIC}

The Kullback-Leibler (KL) information is denoted by $K L(g: f)$, and the random variables $X, U$ have the same distribution as the probability model for $\boldsymbol{X}_{\boldsymbol{t}}$. Then $K L(g: f)$ is given by

$$
\begin{aligned}
& K L(g: f)=\int_{0}^{\infty} \log \frac{g\left(x \mid \boldsymbol{\theta}_{o}\right)}{f(x \mid \boldsymbol{\theta})} g\left(x \mid \boldsymbol{\theta}_{o}\right) d x \\
& =\int_{0}^{\infty} \log g\left(x \mid \boldsymbol{\theta}_{o}\right) g\left(x \mid \boldsymbol{\theta}_{o}\right) d x \\
& -\int_{0}^{\infty} \log f(x \mid \boldsymbol{\theta}) g\left(x \mid \boldsymbol{\theta}_{o}\right) d x,
\end{aligned}
$$

where $g\left(x \mid \boldsymbol{\theta}_{o}\right)$ is the probability density function for the true model, and $f(x \mid \boldsymbol{\theta})$ is the probability density function for the fitted model. When $K L(g: f) \geq 0$ is close to 0 , the fitted model means to be close to the true model.

The positive term of $K L(g: f)$ is unknown, but the negative term means $n$ times the expected mean log likelihood which is denoted by $E L L$ and given by

$$
\begin{aligned}
& E L L=E_{X}\left\{E_{U}\{\log l(\hat{\boldsymbol{\theta}}(\boldsymbol{x}) \mid D \boldsymbol{u})\}\right\} \\
& =n E_{X}\left\{\int_{0}^{\infty} \log f(u \mid \hat{\boldsymbol{\theta}}(\boldsymbol{x})) g\left(u \mid \boldsymbol{\theta}_{o}\right) d u\right\} .
\end{aligned}
$$

However $\log l\left(\hat{\boldsymbol{\theta}}\left(\boldsymbol{X}_{\boldsymbol{t}}\right) \mid D_{\boldsymbol{X}_{\boldsymbol{t}}}\right)$ is an estimate of ELL, then the bias correction is necessary to the estimation of ELL. EIC consists of the bias correction based on the data $D_{\boldsymbol{X}_{t}}$ and the bootstrap samples, and the bias $C^{*}$ is given by

$$
\begin{aligned}
C^{*}=E_{X^{*}} & \left\{\log l\left(\hat{\boldsymbol{\theta}}_{A}\left(\boldsymbol{x}^{*}\right) \mid D_{\boldsymbol{x}^{*}}\right)-l\left(\hat{\boldsymbol{\theta}}_{A}\left(\boldsymbol{X}_{\boldsymbol{t}}\right) \mid D_{\boldsymbol{x}^{*}}\right)\right\} \\
+\quad E_{X^{*}} & \left\{E_{U^{*}}\left\{\log l\left(\hat{\boldsymbol{\theta}}_{A}\left(\boldsymbol{X}_{\boldsymbol{t}}\right) \mid D_{\boldsymbol{u}^{*}}\right)\right\}\right. \\
-E_{U^{*}} & \left.\left\{\log l\left(\hat{\boldsymbol{\theta}}_{A}\left(\boldsymbol{x}^{*}\right) \mid D \boldsymbol{u}^{*}\right)\right\}\right\},
\end{aligned}
$$

where $X^{*}, U^{*}$ are bootstrap samples and $\hat{\boldsymbol{\theta}}_{A}\left(\boldsymbol{X}_{\boldsymbol{t}}\right)$ is any estimator based on the data $D_{\boldsymbol{X}_{\boldsymbol{t}}}$. In this paper, the bootstrap samples are driven by the fitted model and estimated parameters under $D_{\boldsymbol{X}_{\boldsymbol{t}}}$. EIC is defined as

$$
E I C=-2 * \times \log l\left(\hat{\boldsymbol{\theta}}_{A}\left(\boldsymbol{X}_{\boldsymbol{t}}\right) \mid D_{\boldsymbol{X}_{\boldsymbol{t}}}\right)+2 \times C^{*} .
$$


In the Bayesian methodologies, the values of EIC are calculated based on the marginal likelihood functions. However, the hybrid of MCMC and the bootstrap methods needs an enormous amount of repetition in the numerical calculation for EIC. Therefore, in this paper, the Laplace's method is also used for the application of EIC with MCMC method.

\section{Concluding Remarks}

In this paper, the stochastic process models have been applied to the degradation evaluation for reliability. In particular, the Lévy process has been emphasized as the central role of the generating degradation models. The estimation methods for the models have been represented and the methodologies have been realized in detail. We have proposed the application EIC to the models with the estimations. It has been shown that the reliability analysis for the degradation is effective based on the theoretical consideration.

\section{References}

[1] Singpurwalla, N. D., Survival in dynamic environments, Statistical Science, Vol.10, No.1, pp.86-103, 1995.

[2] Abdel-Hameed, M., Degradation process: an overview, Advanced in Degradation Modeling, Statistics for Industry and Technology, Nikulin, M. S., et.al. eds., pp.17-25, Birkhäuser, 2010.

[3] Wang, X. and Xu, D., An inverse Gaussian process model for degradation data, Technometrics, Vol.52, No.2, pp.188-197, 2010.

[4] Padgett, W. J. and Tomlinson, M. A., Inference from accelerated degradation and failure data based on Gaussian process models, Lifetime Data Analysis, Vol.10, pp.191-206, 2004.

[5] Park, C. and Padgett, W. J., Accelerated degradation models for failure based on geometric Brownian motion and gamma processes, Lifetime Data Analysis, Vol.11, pp.511-527, 2005.

[6] Basu, S. and Lingham, R. T., Bayesian estimation of system reliability in Brownian stress-strength models, Annals of the Institute of Statistical Mathematics, Vol.55, No.1, pp.7-19, 2003.

[7] Konishi, S. and Kitagawa, G., Information Criteria and Statistical Modeling, Springer, 2008.

[8] Iacus, S. M., Simulation and Inference for Stochastic Differential Equation, Springer, 2008.

[9] Tierney, L., and Kadane, J. B., Accurate approximations for posterior moments and marginal densities, Journal of the American Statistical Association, Vol.81, pp.81-86,1986.
[10] Meeker, W. Q. and Escobar, L. A., Statistical Methods for Reliability Data, pp.316342,Wiley, 1998.

[11] Lu, C. J. and Meeker, W. Q.,Using degradation measures to estimate a time-to-failure distribution, Technometrics, Vol.35, No.2,pp.161-174,1993. 